\title{
Erratum to: Probiotic treatments for induction and maintenance of remission in inflammatory bowel diseases: a meta-analysis of randomized controlled trials
}

\author{
Mikihiro Fujiya $\cdot$ Nobuhiro Ueno $\cdot$ Yutaka Kohgo
}

Published online: 29 January 2014

(C) Springer Japan 2014

Erratum to: Clin J Gastroenterol

DOI 10.1007/s12328-013-0440-8

In Table 1, the words "High" and "Low" were transposed. All occurrences of "High" should be "Low" and vice versa. The correct table is shown as follows.

The online version of the original article can be found under doi: 10.1007/s12328-013-0440-8.

M. Fujiya $(\bowtie) \cdot$ N. Ueno $~ Y$ Y. Kohgo

Division of Gastroenterology and Hematology/Oncology,

Department of Medicine, Asahikawa Medical University, 2-1

Midorigaoka-higashi, Asahikawa, Hokkaido 078-8510, Japan

e-mail: fjym@asahikawa-med.ac.jp 
Table 1 The risk of bias in the included studies

\begin{tabular}{|c|c|c|c|c|c|c|}
\hline References & $\begin{array}{l}\text { Random sequence } \\
\text { generation }\end{array}$ & $\begin{array}{l}\text { Allocation } \\
\text { concealment }\end{array}$ & $\begin{array}{l}\text { Blind participants, personnel } \\
\text { and outcome assessors } \\
\text { of outcome }\end{array}$ & Incomplete outcome data & $\begin{array}{l}\text { Selective } \\
\text { reporting }\end{array}$ & $\begin{array}{l}\text { Jadad } \\
\text { score }\end{array}$ \\
\hline$[55]$ & Unclear & Unclear & Low & Low & Low & 4 \\
\hline$[51]$ & Unclear & Unclear & High & Low & Low & 3 \\
\hline$[62]$ & Low & Low & Low & Low & Low & 5 \\
\hline [59] & Low & Low & Low & Low & Low & 5 \\
\hline$[60]$ & Low & Low & Low & Low & Low & 5 \\
\hline [48] & Unclear & Unclear & Low & High & Low & 3 \\
\hline$[52]$ & Unclear & Unclear & High & Low & Low & 3 \\
\hline$[56]$ & Unclear & Unclear & Low & Low & Low & 4 \\
\hline$[50]$ & Low & Low & Low & Low & Low & 5 \\
\hline$[63]$ & Unclear & Unclear & Low & Low & Low & 5 \\
\hline$[57]$ & Unclear & Unclear & High & Low & Low & 3 \\
\hline$[64]$ & Low & Low & Low & Low & Low & 5 \\
\hline [65] & Low & Low & Low & Low & Low & 5 \\
\hline [53] & Low & Low & Low & Low & Low & 5 \\
\hline [46] & Low & Low & Low & Low & Low & 5 \\
\hline [54] & Low & Low & Low & Low & Low & 5 \\
\hline [47] & Low & Low & Low & Low & Low & 5 \\
\hline$[61]$ & Low & Low & Low & Low & Low & 5 \\
\hline [58] & Low & Low & High & Low & Low & 4 \\
\hline [49] & Low & Low & Low & Low & Low & 5 \\
\hline
\end{tabular}

